\title{
Design, Modeling and Control of a Soft Robotic Arm
}

\section{Conference Paper}

Author(s):

Hofer, Matthias; D'Andrea, Raffaello

Publication date:

2018

Permanent link:

https://doi.org/10.3929/ethz-b-000296041

Rights / license:

In Copyright - Non-Commercial Use Permitted

Originally published in:

https://doi.org/10.1109//ROS.2018.8594221 


\title{
Design, Modeling and Control of a Soft Robotic Arm
}

\author{
Matthias Hofer and Raffaello D'Andrea
}

\begin{abstract}
In this paper we present the design of a hybrid robotic arm using soft, inflatable bladders for actuation. Low cost switching valves are used for pressure control, where the valve model is identified experimentally. A model of the robotic arm is derived based on system identification and used to derive a linear quadratic Gaussian controller. A method to solve limitations of the employed switching valves is proposed and experimentally proven to improve tracking performance. The closed loop control performance of the robotic arm is demonstrated by stabilizing a rotational inverted pendulum known as the Furuta pendulum.
\end{abstract}

\section{INTRODUCTION}

Soft inflatable actuators are attracting increasing interest for a number of reasons: They can actuate quickly [1], have a high payload to weight ratio [2], high power density [3], are low cost [4], and are nevertheless safe to interact with humans [5], [6]. As a consequence of their low inertia and high compliance, they can be employed side by side with humans or even physically interact with them.

A comprehensive overview of soft inflatable actuators is provided in [7] and [8]. Soft inflatable actuators can be grouped based on the material from which they are built. A general distinction can be made between molded elastomer type actuators and actuators built from fabrics. In this work, we focus on the second type of actuators and employ them to control a robotic arm with one degree of freedom (DoF).

Different design paradigms exist for inflatable manipulators that vary in the degree of softness. The authors of [9] propose an entirely soft inflatable manipulator, where the actuator, supporting structure and joints are all made from fabric. Using only fabric reduces the inertia and consequently the risk of causing damage, but introduces a high number of potentially uncontrollable degrees of freedom.

Using fabric along with some rigid parts to lock passive degrees of freedom can improve control authority and consequently simplify the control task. A combination of a rigid joint with an inflatable actuator and link to improve positioning accuracy is described in [10]. A hybrid system where inflatable bladders are supported by a spine-like structure to control the bending behavior is proposed in [11]. An assistance robot with a rotary elastic chamber actuator combined with rigid links is presented in [12]. In all the above mentioned examples, the use of rigid components leads to an increase in inertia and decreases compliance of the overall manipulator. The ideal trade-off between entirely soft robots and hybrid designs most probably depends on the specific application.

The authors are members of the Institute for Dynamic Systems and Control, ETH Zürich, Switzerland. \{hofermat, rdandrea\} @ethz . ch

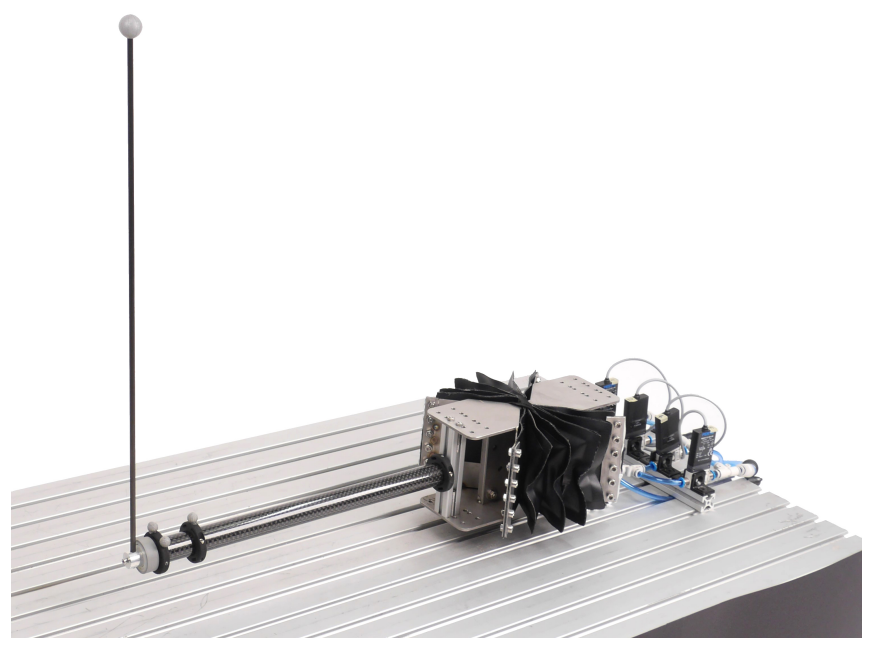

Fig. 1. The hybrid robotic arm described in this paper balancing a Furuta pendulum. Two soft inflatable bladders (in black) actuate a one DoF robotic arm made from rigid components.

Two design approaches exist for inflatable actuators (and inflatable links). They can consist of a single layer of fabric, which has to be airtight and absorb the tensile stress (see e.g. [13]) or two layers (see e.g. [9]), where the inner layer is airtight and the outer layer absorbs the forces. The single layer design has the advantage of simpler manufacture, while the double layer configuration allows separation of the requirements of airtightness and tensile strength into two separate materials. Different combinations of airtight polyurethane as an inner layer with reinforcement fabrics in the outer layer are discussed in [14]. Depending on the material employed, different fabrication methods apply. The most common manufacturing techniques are sewing, heat and high frequency welding.

The modeling of inflatable actuators is a challenging and active field of research. Soft materials exhibit visco-elastic material behavior, which can cause hysteresis effects. A static mechanical model to describe the bending of inflatable links is derived in [15]. Pressure dependent torque, stiffness and damping maps of the actuator are identified in [16]. A rigid body inspired model is used in [17], where parameters are determined experimentally. Finite element methods are applied in [18] to investigate the material response of both actuator and structural components.

The intrinsic compliance of an inflatable manipulator and its material complexity make accurate position control challenging. Different control approaches have been investigated 
for soft inflatable actuators. In [19] proportional-integral control is applied to a two DoF system, whereas the authors of [20] use a sliding mode control approach. The authors of [9] demonstrate simultaneous position and stiffness control for an inflatable robotic arm with antagonistically arranged actuators. In [21] model predictive control is proposed to improve position tracking performance with a pneumatically actuated humanoid.

In this paper we present the design of a hybrid robotic arm consisting of soft, inflatable actuators and a rigid joint and link (see Fig. 1). Two antagonistically arranged bellow-type actuators control the angle of a one DoF manipulator. The actuators are made from a single layer of high frequency weldable fabric, which allows for high actuation pressures. Four low-cost switching valves are used for pressure control and an approach to reduce limitations of these valves is presented. The closed loop control performance of the robotic arm is demonstrated by balancing a Furuta pendulum [22].

The remainder of this paper is organized as follows: The design of a soft actuator and the hybrid robotic arm are described in Section II. A model of the pressure and arm dynamics is derived in Section III and a cascaded control architecture for both subsystems is presented in Section IV. Results from pressure tracking experiments and the stabilization of the Furuta pendulum are presented in Section V. A conclusion is drawn in Section VI.

\section{DESIGN}

The design of a hybrid soft robotic arm is presented in this section. The arm consists of two soft inflatable actuators (Fig. 2), a rigid one DoF joint and a rigid link. The soft actuator is described in the first part of this section and the complete robotic arm in the second part.

\section{A. Soft Inflatable Actuator}

We developed a bellow-type actuator consisting of six single cushions made from fabric. The inner seam, which connects two cushions, is placed off-center. This causes the actuator to expand in the angular direction when pressurized, which is used to generate a rotary motion. The actuator consists of a single layer of thermoplastic polyurethane

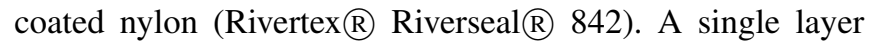
design simplifies manufacturing, but increases the requirements of the material employed. The fabric has to be airtight and additionally requires high tensile strength to withstand the actuation pressure. The material can be processed with high frequency welding, a manufacturing process where a high frequency electromagnetic field is applied to weld two sheets of polyurethane-coated nylon together. High frequency welding differs from similar manufacturing techniques, such as thermal welding, in the way the heat is applied to the fabric. While the heat is provided from the outside in thermal welding, it is generated over the full thickness of the seam in high frequency welding. This is achieved by an excitation of the polymer at a molecular level (more details can be found in [23]). The result is a high seam quality allowing for high actuation pressures of up to 4.5 bar for the actuator discussed

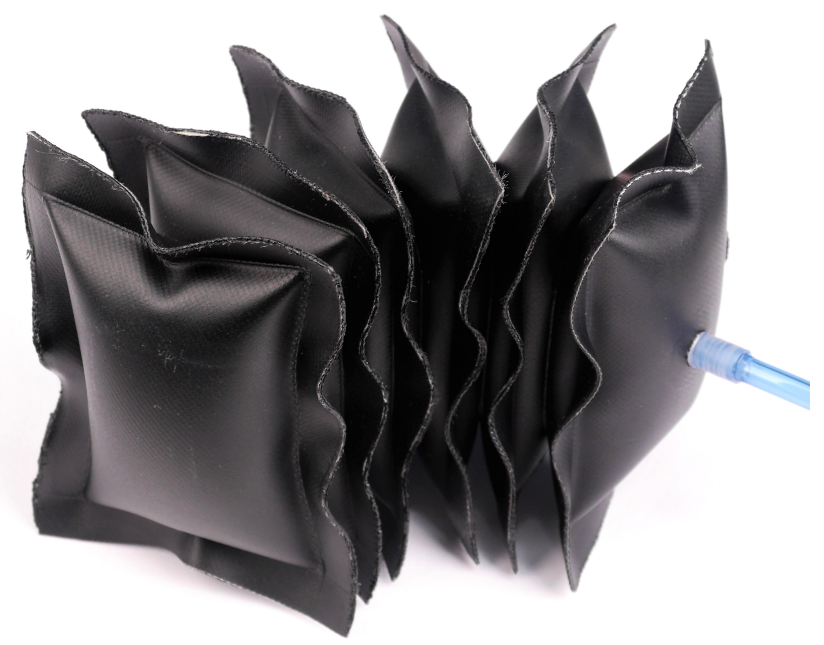

Fig. 2. A fully inflated bellow-type actuator consisting of six single cushions made from polyurethane coated nylon. The front side measures $110 \times 90 \mathrm{~mm}$ in a deflated state and the maximum actuation range, when fully inflated, is approximately $110^{\circ}$. A large seam width of $10 \mathrm{~mm}$ allows for high forces and hence high actuation pressures. The inner seam combining two cushions is rounded at its corners to reduce stress peaks. A tube connects the actuator to the pressure sensor and the control valves.

in this work. Tubing is connected to the bladder through a high frequency weldable polyvinyl chloride tubing flange.

The pressure is measured and controlled for each actuator separately. In general, two types of valves for pressure control exist. Proportional solenoid valves have a continuously adjustable nozzle and therefore, allow accurate adjustment of the pressure. Switching solenoid valves on the other hand only have two discrete states, namely fully open or fully closed. Continuous control of the air flow through the valve and consequently pressure is realized by means of pulse width modulation (PWM). We use 2/2-way switching valves (two connections/two switching positions; Festo MHJ10) which are considerably less expensive than proportional valves. Two switching valves are used per actuator and are 3.5 times less expensive than one proportional valve of the same manufacturer. Three pressure sensors are used to measure the pressure in both actuators and at the source. A pneumatic diagram of the system is depicted in Fig. 3.

\section{B. Robotic Arm}

Two soft actuators are arranged antagonistically around a rigid one DoF revolute joint. The angular expansion of both actuators generates a torque, which is used to control the rotary motion of the robotic arm. The torque generated depends on the pressure difference between the two bladders. A rigid link made from carbon fiber is attached to a metal joint. The first and last cushions of each actuator are clamped to the joint. A strap is arranged around both actuators to concentrate the inflation-induced expansion of the actuator in the angular direction and limit expansion in the radial direction.

The Furuta pendulum is attached to the tip of the robotic arm and can pivot about the arm axis. Hence, its motion is 


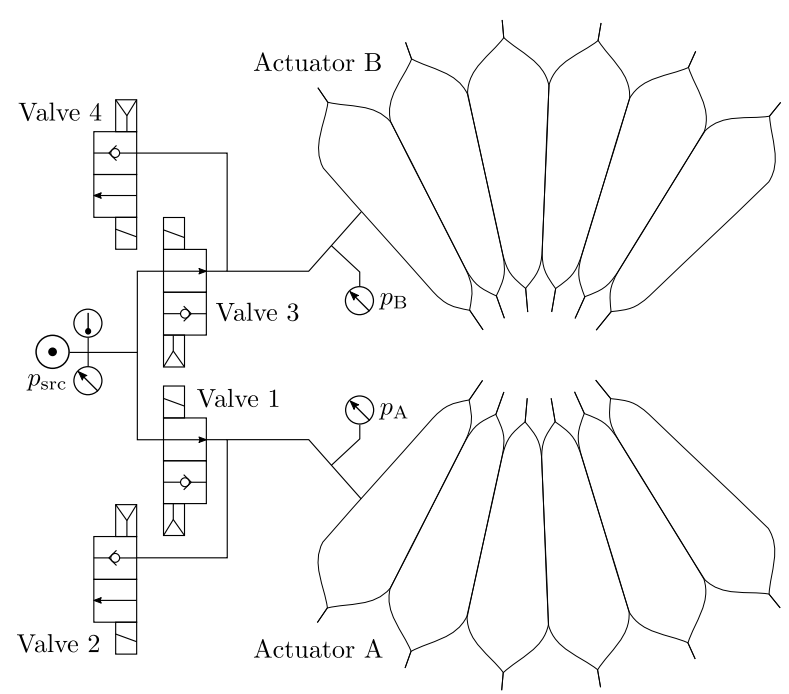

Fig. 3. Pneumatic diagram of the pressure system consisting of two actuators, four $2 / 2$-way switching valves, three pressure sensors and one temperature sensor. Valves 1 and 3 are connected to a pressure source and used to increase the pressure in the bladder, while valves 2 and 4 are connected to ambient pressure and used to decrease pressure. The pressure is measured in both actuator $p_{\mathrm{A}}, p_{\mathrm{B}}$ and at the source $p_{\text {src }}$, where we also measure the temperature.

restricted to the plane perpendicular to the axis of the arm. The pendulum is made from carbon fiber and considerably lighter than the robotic arm.

\section{Modeling And System Identification}

The dynamics of the hybrid robotic arm can be broken down into the pressure dynamics of the actuator and the motion dynamics of the arm. In the first part of this section, a first principle model of the pressure dynamics is introduced and a static model of the valve is derived from experimental data. In a second step, the robotic arm dynamics are identified using a system identification procedure. A first principles model is used to describe the dynamics of the Furuta pendulum, which is then combined with the arm dynamics in a linear time invariant (LTI) model. For the ease of notation vectors are expressed as n-tuples with dimension and stacking clear from the context.

\section{A. Valve and Pressure Dynamics}

The objective is to control the pressure in actuators A and B. This is directly affected by the mass flow in or out of the actuator, which in turn depends on the duty cycle applied to the valves. The pressure dynamics of a single actuator can be described by a gray box model derived in [24],

$$
\dot{m}=\frac{1}{R T}\left(\frac{V \dot{p}}{\kappa}+p \dot{V}\right),
$$

where $\dot{m}$ is the mass flow, $R$ the ideal gas constant, $T$ the temperature, $\kappa$ denotes the polytropic coefficient and $V$, $\dot{V}$ are (approximations of) the volume and volume change, respectively. The polytropic coefficient satisfies $\kappa \in[1,1.4]$, where the lower limit corresponds to an isothermal process and the upper limit to an adiabatic process. The underlying assumptions are perfect gas, mass and energy conservation, a polytropic process and negligible kinetic and potential energy changes. Additionally, the elasticity of the fabric material and interactions with the antagonistic actuator are neglected. The volume is approximated by a second order polynomial function of the angle, where coefficients are identified experimentally. Computing the time derivative of the volume yields an expression for the volume change as a function of the angle and angular velocity.

Next, we derive a static model for the mass flow as a function of the pressure ratio over the valve and the applied duty cycle. When considering a fully opened valve, the mass flow can be described by the ISO 6358 standard model [25],

$$
\begin{aligned}
& \dot{m}_{\mathrm{fo}}\left(p_{\mathrm{u}}, p_{\mathrm{d}}\right)= \\
& \begin{cases}p_{\mathrm{u}} C \rho_{0} \sqrt{T_{0} / T_{\mathrm{u}}} \sqrt{1-\frac{\left(\frac{p_{\mathrm{d}}}{p_{\mathrm{u}}}-b\right)}{(1-b)}} & \text { if } \frac{p_{\mathrm{d}}}{p_{\mathrm{u}}}>b \\
p_{\mathrm{u}} C \rho_{0} \sqrt{T_{0} / T_{\mathrm{u}}} & \text { if } \frac{p_{\mathrm{d}}}{p_{\mathrm{u}}} \leq b,\end{cases}
\end{aligned}
$$

where $\dot{m}_{\mathrm{fo}}$ stands for the mass flow of the fully opened valve and the arguments $p_{\mathrm{u}}, p_{\mathrm{d}}$ denote up and downstream pressure, respectively. The parameters $\rho_{0}, T_{0}$ denote density and temperature at the ISO 6358 reference state, $b$ the critical pressure ratio which determines a choked flow condition and $C$ the sonic conductance. The parameters $b$ and $C$ are typically provided by the valve manufacturer.

The mass flow of a fully opened valve, $\dot{m}_{\mathrm{fo}}$, represents the maximum possible mass flow for a given pressure ratio over the valve and is scaled down when applying a pulse width modulation with a duty cycle of less than one. The dependency on the duty cycle is investigated in static identification experiments. A mass flow sensor is used to measure the mass flow through a valve for different duty cycles and pressure ratios. We follow a similar procedure to that described in [26]. The duty cycle is set directly, while the pressure ratio is adjusted by changing the upstream source pressure and enforcing ambient pressure conditions at the valve outlet. Up and downstream pressures are recorded and a PWM frequency of $200 \mathrm{~Hz}$ is used throughout the experiments. The measured mass flow, $\dot{m}$ is plotted in Fig. 4 over the duty cycle and the maximum possible mass flow of a fully opened valve, $\dot{m}_{\text {fo }}$. Note that the pressure ratio over the valve is incorporated in $\dot{m}_{\mathrm{fo}}$ as defined in (2).

Three observations can be made. First, the measured mass flow (black dots) for a duty cycle of one is accurately described by the standard model and consequently coincides with $\dot{m}_{\text {fo }}$. Second, the measured mass flow generally decreases smoothly with the decreasing duty cycle. Finally, a discontinuity is present for small duty cycles, where the mass flow is zero. This behavior can be described with a bi-linear model of the duty cycle and $\dot{m}_{\mathrm{fo}}$ combined with a dead band region for small duty cycles,

$$
\dot{m}= \begin{cases}d c \cdot \dot{m}_{\mathrm{fo}} & \text { if }\left(d c, \dot{m}_{\mathrm{fo}}\right) \notin \mathcal{S}_{\mathrm{db}} \\ 0 & \text { otherwise },\end{cases}
$$

where $\mathcal{S}_{\mathrm{db}}$ denotes the dead band. The model identified and described by (3) is depicted in Fig. 4. It captures the principal 


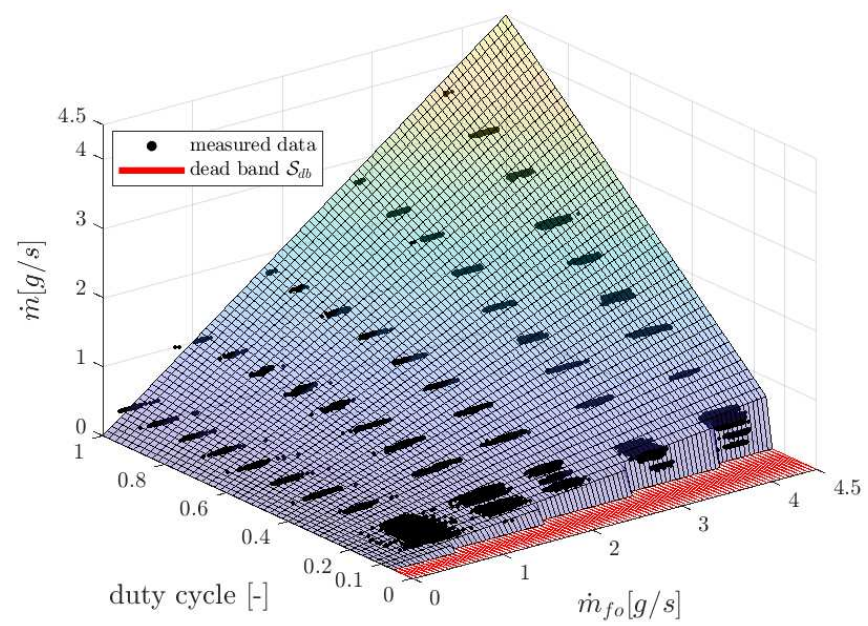

Fig. 4. The identified static model of the valve. The mass flow through the valve, $\dot{m}$, as a function of the applied PWM duty cycle and the maximum possible mass flow of a fully opened valve $\dot{m}_{\text {fo }}$. The mass flow predicted by the model is depicted in blue to orange, while the measured data points are depicted in black. The duty cycle is increased in steps of 0.01 for small values and 0.1 for values above 0.2 . The red region depicts the dead band present for small duty cycles, where the valve opening time is insufficient for a non-zero mass flow. The resolution of the grid points to which the model is applied is reduced for the sake of readability. However, the boundary of the dead band is a straight line in the $x-y$ plane.

behavior, but slightly underestimates the mass flow for small values of $\dot{m}_{\mathrm{fo}}$ and overestimates the mass flow for large values of $\dot{m}_{\text {fo }}$. Note that because a higher upstream pressure is acting on the valve nozzle for higher $\dot{m}_{\mathrm{fo}}$, the dead band increases as well.

\section{B. Robotic Arm Dynamics}

The dynamics of the robotic arm are identified without the pendulum being attached to the arm. The robotic arm has a significantly higher inertia than the pendulum and it is thus assumed that the effect of the pendulum on the arm dynamics is negligible. The dynamics of the pendulum are derived in the third part of this section.

The pressure difference between the bladders, $\Delta p=p_{\mathrm{A}}-$ $p_{\mathrm{B}}$ is chosen as the input to the rigid body dynamics of the robotic arm, where a positive value of $\Delta p$ accelerates the arm in the positive $\alpha$-direction (see Fig. 5). For example, the arm can generate a torque of $11 \mathrm{Nm}$ at its center position $(\alpha=0)$, when a pressure difference of 0.8 bar is applied.

The dynamics of the robotic arm are identified using a black box system identification procedure. The advantage of this approach is its simplicity. It can be quickly repeated after a design change and does not require measurement campaigns to determine e.g. the angle dependent pressuretorque characteristic. The transfer function from the input $\Delta p$ to the angular orientation $\alpha$ is a single-input single-output system. Note that we derive the dynamics from the actual pressure difference and not its set point.

The system is excited by a signal of superposed sinusoids with frequencies in the range of $[0.1,4 \mathrm{~Hz}]$. The amplitudes are chosen such that non-linearities close to the actuation

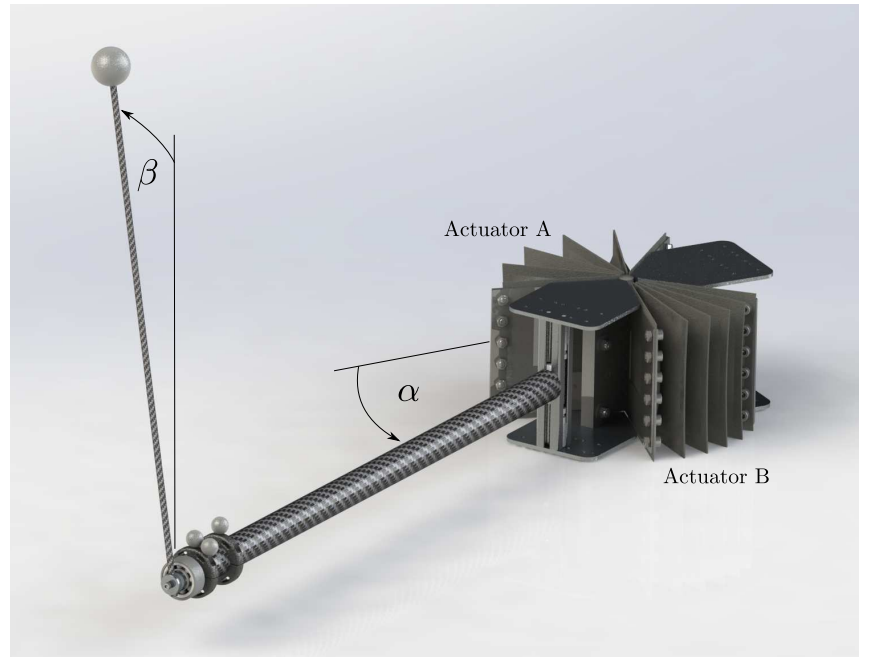

Fig. 5. Illustration of the one DoF robotic arm with the Furuta pendulum attached. The arm angle relative to its center position is denoted by $\alpha$ and the pendulum deflection by $\beta$.

limits of the arm are not excited. The Crest factor is minimized by choosing the phases of the sinusoids as the Newman phases [27]. The initial transient response of the output is discarded and the twelve consecutive repetitions are averaged in order to improve the signal to noise ratio. A continuous time second order model including a delay is assumed for the arm dynamics,

$$
G(s)=\frac{\alpha(s)}{\Delta p(s)}=k \frac{\omega_{0}^{2}}{\omega_{0}^{2}+2 \delta \omega_{0} s+s^{2}} \cdot e^{-T s},
$$

with $s$ denoting the complex variable. The coefficients are identified from the measured transfer function as $k=1.86$, $\omega_{0}=22.36, \delta=0.40, T=0.006$. The resulting fit is depicted in Fig. 6.

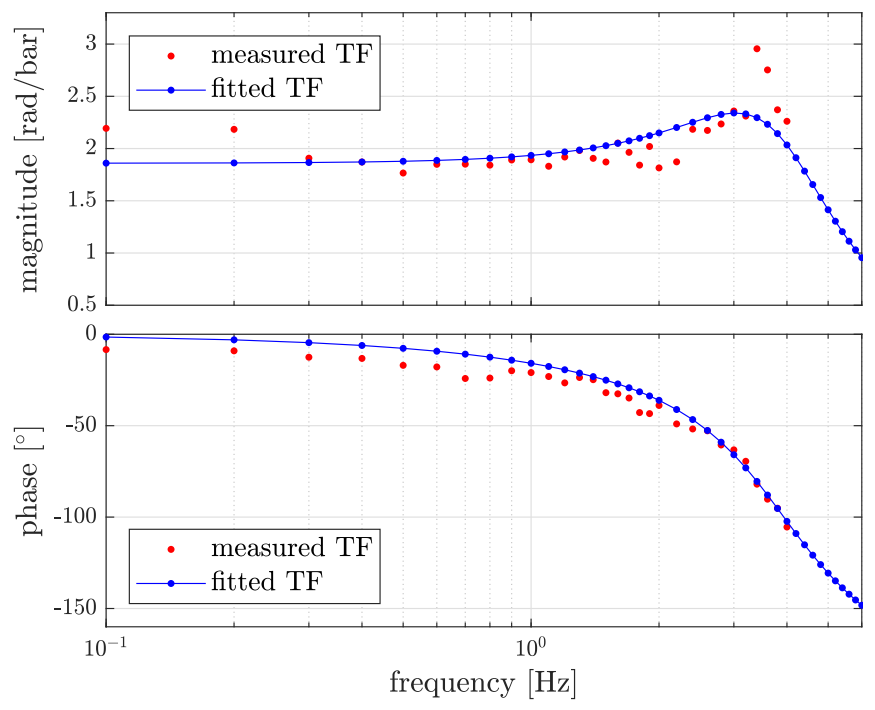

Fig. 6. Bode plot of the measured and fitted second order transfer function of the arm dynamics from pressure difference $\Delta p$ (in bar) to angular orientation $\alpha$ (in rad). The second order model underestimates the magnitude for small frequencies and the identified value of the natural frequency indicates the fast dynamics of the arm. 
The delay can be neglected since its effect on the phase is not relevant for the bandwidth considered. Applying the inverse Laplace transformation gives a linear differential equation for the robotic arm dynamics

$$
\frac{1}{\omega_{0}^{2}} \ddot{\alpha}=-\alpha-\frac{2 \delta}{\omega_{0}} \dot{\alpha}+k \Delta p .
$$

Equation (5) will be used in the next part of this section to derive an LTI system of the robotic arm combined with the Furuta pendulum.

\section{Furuta Pendulum Dynamics}

The pendulum deflection is denoted by $\beta$ (see Fig. 5) and the complete dynamics of the Furuta pendulum are derived in [22]. It is assumed that the principle moment of inertia in the longitudinal direction of the pendulum is negligible and the principle moments of inertia in the lateral direction with respect to the joint are equal and denoted by $J_{2}$. The dynamics can then be described by

$$
\begin{aligned}
J_{2} \ddot{\beta} & =L_{1} l_{2} m_{2} \cos (\beta) \ddot{\alpha}+\frac{1}{2} J_{2} \sin (2 \beta) \dot{\alpha}^{2} \\
& +g l_{2} m_{2} \sin (\beta)-d_{2} \dot{\beta} .
\end{aligned}
$$

The physical properties of the pendulum are explained and summarized in Table I. Note that the pendulum dynamics are linked via $\ddot{\alpha}$ and $\dot{\alpha}$ in (6) to the arm dynamics.

TABLE I

Physical Parameters of the Robotic Arm and Pendulum

\begin{tabular}{lll}
\hline & Value & Description \\
\hline$d_{2}$ & $1 \mathrm{e}-5 \mathrm{Nms} / \mathrm{rad}$ & Viscous friction of pendulum \\
$g$ & $9.81 \mathrm{~m} / \mathrm{s}^{2}$ & Gravitational acceleration \\
$J_{2}$ & $0.0017 \mathrm{kgm}^{2}$ & Inertia of pendulum with respect to joint \\
$L_{1}$ & $0.45 \mathrm{~m}$ & Length of arm \\
$L_{2}$ & $0.48 \mathrm{~m}$ & Length of pendulum \\
$l_{2}$ & $0.32 \mathrm{~m}$ & Dist. from joint to center of mass of pendulum \\
$m_{2}$ & $0.014 \mathrm{~kg}$ & Mass of pendulum \\
\hline
\end{tabular}

Replacing $\ddot{\alpha}$ by (5) and linearizing the dynamics around $(\alpha, \dot{\alpha}, \beta, \dot{\beta})=(0,0,0,0)$ yields the LTI system

$$
\left(\begin{array}{l}
\dot{x}_{1} \\
\dot{x}_{2} \\
\dot{x}_{3} \\
\dot{x}_{4}
\end{array}\right)=\underbrace{\left(\begin{array}{cccc}
0 & 10 & 0 & 0 \\
-50 & -18 & 0 & 0 \\
0 & 0 & 0 & 10 \\
-57.18 & -20.59 & 2.47 & -0.01
\end{array}\right)}_{:=\mathrm{A}}\left(\begin{array}{l}
x_{1} \\
x_{2} \\
x_{3} \\
x_{4}
\end{array}\right)+\underbrace{\left(\begin{array}{c}
0 \\
29.59 \\
0 \\
33.84
\end{array}\right)}_{:=\mathrm{B}} u .
$$

The state $x$ corresponds to $(\alpha, \dot{\alpha}, \beta, \dot{\beta})$ normalized by $(\pi, 10 \pi, \pi, 10 \pi)$ and the control input $u$ to the pressure difference $\Delta p$ (in $\mathrm{Pa}$ ) normalized by $\bar{p}=1 e 5$. The arm angle $\alpha\left(x_{1}\right)$ and the pendulum deflection $\beta\left(x_{3}\right)$ are directly measurable (see Section V), leading to the following measurement equation,

$$
\left(\begin{array}{l}
y_{1} \\
y_{2}
\end{array}\right)=\underbrace{\left(\begin{array}{llll}
1 & 0 & 0 & 0 \\
0 & 0 & 1 & 0
\end{array}\right)}_{:=\mathrm{C}}\left(\begin{array}{l}
x_{1} \\
x_{2} \\
x_{3} \\
x_{4}
\end{array}\right)+\underbrace{\left(\begin{array}{l}
0 \\
0
\end{array}\right)}_{:=\mathrm{D}} u .
$$

\section{CONTROL}

We present a cascaded control architecture based on time scale separation of the faster pressure dynamics in an inner loop and the slower arm/pendulum dynamics in an outer loop (see Fig. 7). It is assumed that the inner control loop can track set point changes instantaneously and without any dynamics. A linear quadratic Gaussian (LQG) controller is used to stabilize the pendulum [28]. It computes pressure set points, which are then tracked for each actuator by a separate pressure controller. The design of the pressure controller is discussed in the first part of this section and the controller for stabilizing the Furuta pendulum in the second part.

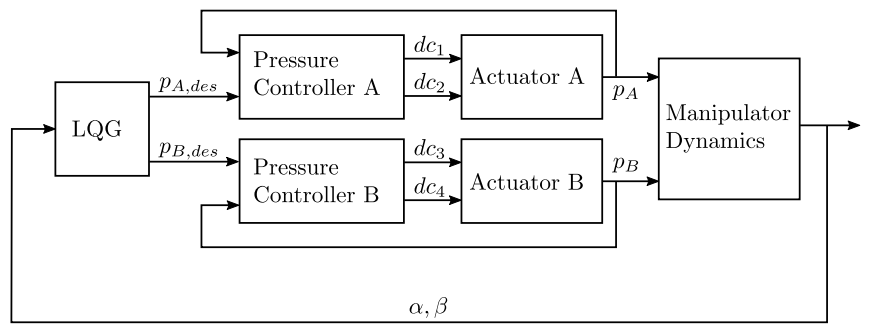

Fig. 7. Cascaded control architecture with an LQG controller in the outer loop running at $50 \mathrm{~Hz}$ and a pressure controller for each actuator in the inner loop running at $200 \mathrm{~Hz}$. The LQG controller outputs the desired pressures of the actuators, which are the set points of the pressure controllers.

\section{A. Pressure Control}

The measured pressures are filtered with a low pass filter $(-3 \mathrm{~dB}$ bandwidth of $32 \mathrm{~Hz}$ ) to smooth the effects of the pulse width modulation and avoid valve jitter. Each pressure controller runs at $200 \mathrm{~Hz}$. The control algorithm is described for actuator A, but is analogously implemented for actuator B. Using loop shaping, we design the control action such that the pressure dynamics behave as

$$
\dot{p}_{\mathrm{A}}=\frac{1}{\tau_{\mathrm{p}}}\left(p_{\mathrm{A}, \mathrm{des}}-p_{\mathrm{A}}\right) .
$$

The time constant of the desired pressure dynamics is denoted by $\tau_{p}$ and is used as a tuning parameter for the controller. Combining this expression with the dynamics of the actuator (1), yields an expression for the desired mass flow $\dot{m}_{\mathrm{A}}$ as a function of the current deviation from the pressure set point,

$$
\dot{m}_{\mathrm{A}}=\frac{1}{R T}\left(\frac{V}{\kappa \tau_{\mathrm{p}}}\left(p_{\mathrm{A}, \mathrm{des}}-p_{\mathrm{A}}\right)+p_{\mathrm{A}} \dot{V}\right) .
$$

For a desired mass flow $\dot{m}_{\mathrm{A}}$ and the given current pressure ratio over the valve (hence given $\dot{m}_{\mathrm{A}, \mathrm{fo}}$ ), we then calculate the duty cycle for both the in and outflow valves. Note that the mass flow $\dot{m}_{\mathrm{A} \text {,fo }}$, has to be calculated for both the in and outflow valves with their corresponding up and downstream pressures. A case distinction is made based on the sign of $\dot{m}_{\mathrm{A}}$. For positive flows, we calculate $d c_{1}$ with (3) and set $d c_{2}$ to zero and vice versa for negative flows. However, (3) cannot be inverted in closed form because of the dead band. Therefore, we calculate the duty cycle in two steps. In the 
first step, we neglect the dead band and calculate the duty cycles as

$$
\begin{aligned}
\tilde{d} c_{1} & = \begin{cases}\frac{\dot{m}_{\mathrm{A}}}{\dot{m}_{\mathrm{A}, \mathrm{f}}\left(p_{\mathrm{scc}}, p_{\mathrm{A}}\right)} & \text { if } \dot{m}_{\mathrm{A}}>0 \\
0 & \text { otherwise }\end{cases} \\
\tilde{d} c_{2} & = \begin{cases}\frac{\left|\dot{m}_{\mathrm{A}}\right|}{\dot{m}_{\mathrm{A}, \mathrm{fo}}\left(p_{\mathrm{A}}, p_{0}\right)} & \text { if } \dot{m}_{\mathrm{A}}<0 \\
0 & \text { otherwise. }\end{cases}
\end{aligned}
$$

Note that for valve 1 , the upstream pressure is the source pressure $p_{\text {src }}$ and the downstream pressure is $p_{\mathrm{A}}$, while for valve 2 , the upstream pressure is $p_{\mathrm{A}}$ and the downstream pressure is the ambient pressure $p_{0}$. The duty cycles $\tilde{d} c_{1}$ and $\tilde{d} c_{2}$ incorporate the identified valve model up to the dead band. In order to avoid operating the valves when no flow is possible, which would result in the valves overheating, the final duty cycles are computed as

$$
d c_{1}= \begin{cases}\tilde{d} c_{1} & \text { if }\left(\tilde{d}_{1}, \dot{m}_{\mathrm{A}, \mathrm{fo}}\left(p_{\mathrm{src}}, p_{\mathrm{A}}\right)\right) \notin \mathcal{S}_{\mathrm{db}} \\ 0 & \text { otherwise }\end{cases}
$$

and analogously for $d c_{2}$. Note that we cannot correct for small deviations from the pressure set point and as a consequence, the tracking performance of the pressure controller and the overall robotic arm is degraded.

A method to circumvent this limitation is to introduce a bypass mass flow from the exit of valves 1 and 3 to the environment. By doing this, the mass flow and correspondingly the duty cycle are constrained to lie outside of the dead band. The bypass is adjusted by a manual throttle and chosen to be as small as possible to limit the amount of wasted air, while ensuring a duty cycle outside of the dead band. The bypass mass flow is compensated in the controller by adding the term

$$
\dot{m}_{\text {bypass }}=\rho_{0} \cdot C_{\text {bypass }} \cdot p_{\mathrm{A}},
$$

to (1), where $C_{\text {bypass }}$ is tuned manually.

\section{B. Motion Control}

A LQG controller consisting of a Kalman filter to provide full state information and a linear quadratic regulator is designed to stabilize the pendulum based on the LTI system (A,B,C,D) derived in Section III. From the optimal control input $u$, the desired pressures for each actuator are calculated as,

$$
\begin{aligned}
& p_{\mathrm{A}, \mathrm{des}}=\max \left(p_{0}, p_{0}+\bar{p} \cdot u\right), \\
& p_{\mathrm{B}, \mathrm{des}}=\max \left(p_{0}, p_{0}-\bar{p} \cdot u\right),
\end{aligned}
$$

with $p_{0}$ being the ambient pressure and $\bar{p}$ the normalization constant of $u$. Note that both desired pressures are bounded below by $p_{0}$ since we are not using a vacuum for deflation. Consequently, the desired pressure difference $p_{\mathrm{A} \text {,des }}-p_{\mathrm{B}, \mathrm{des}}$ is given by $\bar{p} \cdot u$. The LTI model employed was derived based on the actual pressure difference, $\Delta p$, while the output of the LQG controller is used as the desired pressure difference, $\Delta p_{\text {des }}$. Therefore, the pressure set points have to be tracked sufficiently well, otherwise the LTI model can deviate considerably.
A common challenge encountered in the design of a controller for the Furuta pendulum is the presence of a limit cycle induced by joint stiction, as for example reported in [29]. A method to counteract this phenomena is to add a high frequency low amplitude jitter signal on top of the control signal to keep the arm moving and thus avoid stiction.

\section{EXPERIMENTAL RESUlts}

In the first part of this section, results from the pressure tracking experiments of the inflatable actuator are presented. The effect of the bypass method to circumvent limitations of the switching valves is discussed. The stabilization of the Furuta pendulum with the robotic arm is outlined in the second part of this section.

The state of the robotic arm and pendulum is estimated from position data provided by an infrared motion capture system running at $200 \mathrm{~Hz}$ resulting in a measurement accuracy of less than one-tenth of a degree. The control algorithms are executed on a dSPACE controller board (DS1104 R\&D). The source pressure is set to 3 bar and the time constant of the pressure controller to $\tau_{\mathrm{p}}=1 / 50$. The polytropic coefficient is set to $\kappa=1$, which corresponds to an isothermal process and conforms well with experimental data. The PWM frequency is set to $200 \mathrm{~Hz}$ as used for the valve identification experiments. The interested reader is referred to the video attachment showing the experimental results.

\section{A. Pressure Tracking}

The pressure tracking performance is evaluated with and without the bypass approach for actuator A. Fig. 8 shows a comparison for different pressure steps. While the bypass approach improves tracking performance for lower pressures, the improvement decreases for higher pressures. An explanation for this behavior could be that (14) underestimates the mass flow for higher pressures, leading to an insufficient compensation of the bypass mass flow and hence the pressure to not exactly reach the setpoint.

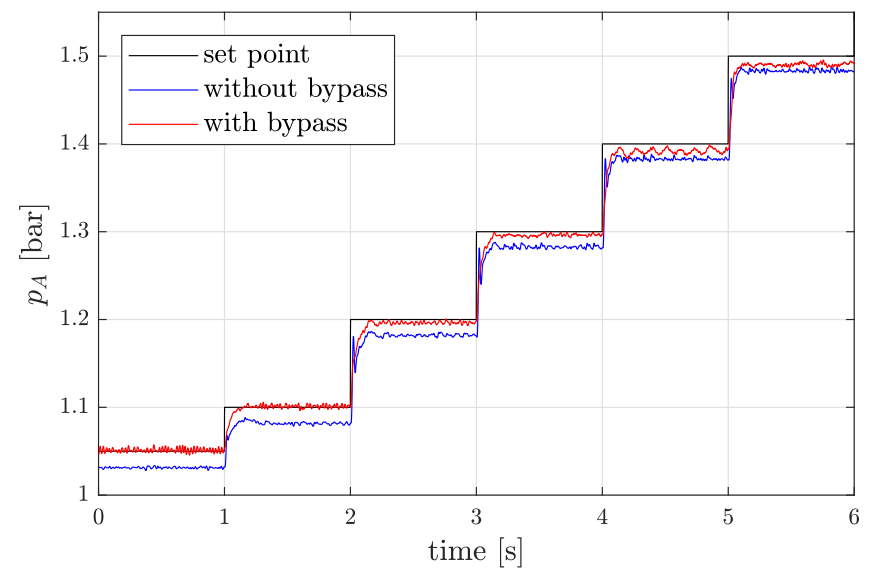

Fig. 8. Results from pressure tracking experiments with actuator A. The set point (in black) increases in steps of 0.1 bar. The blue curve depicts the pressure tracking with the bypass disabled, while the red curve depicts the tracking with the bypass employed. 


\section{B. Stabilization of the Furuta Pendulum}

Zero mean Gaussian noise on both the process and measurement equation with covariance matrices

$$
\begin{aligned}
V & =\operatorname{diag}\left(1,10^{4}, 1,10^{4}\right), \\
W & =\operatorname{diag}(1,1),
\end{aligned}
$$

is assumed. A steady state Kalman filter provides full state information. The cost matrices of the linear quadratic regulator are chosen as

$$
\begin{aligned}
& Q=\operatorname{diag}(20,0.5,100,0.5), \\
& R=10 .
\end{aligned}
$$

Deviations of the pendulum deflection $\beta\left(x_{3}\right)$ are penalized the most and the cost on $\alpha\left(x_{1}\right)$ ensures that the arm remains within its working range. The LQG controller runs at $50 \mathrm{~Hz}$ and the faster poles of the resulting closed loop system are $-22.1 \pm 27.5 i$. The jitter signal for avoiding stiction is chosen as a binary signal switching at $60 \mathrm{~Hz}$ and with an amplitude of 0.04 bar.

The results of the robotic arm balancing the pendulum are depicted in Fig. 9. At $t=\{2,4.7,8 s\}$, a manual disturbance was applied to the pendulum, which can successfully be recovered by the controller. The jitter signal applied helps to reduce the limit cycle of $\alpha$ substantially from $8^{\circ}$ in peak-topeak amplitude without the jitter signal to less than $2^{\circ}$ with the jitter signal, as depicted in the first plot of Fig. 9. The pressure tracking performance when balancing the pendulum was sufficient without using the bypass approach.

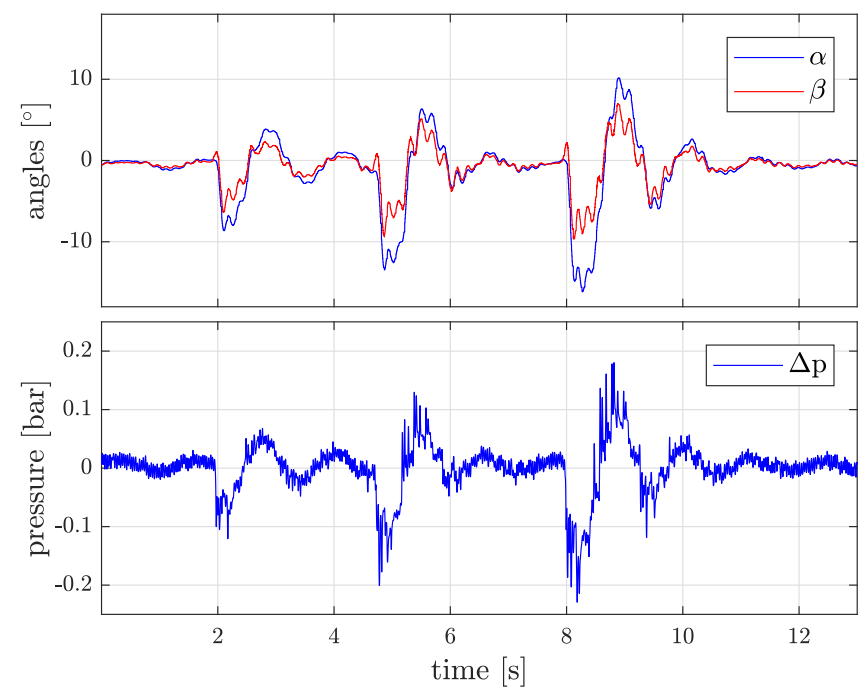

Fig. 9. Experimental results of the robotic arm balancing the Furuta pendulum. The top plot shows the angle of the arm and pendulum and the bottom plot shows the pressure difference. The top plot indicates the presence of a limit cycle in both angles. The high frequency jitter signal on top of the control signal can be seen in the bottom plot.

\section{CONCLUSION}

A hybrid robotic arm using soft inflatable actuators has been presented. A modeling approach based on first principles and system identification techniques has been used to derive a simple model-based controller. A method to reduce the limitations of the low cost switching valves employed has been proposed and proved to improve pressure tracking performance if very precise adjustments are required. The closed loop control performance of the robotic arm has been demonstrated by balancing a Furuta pendulum.

The hybrid design allowed for high positioning accuracy and hence high overall control performance, however, at the expense of higher inertia and reduced compliance. A reduction of the inertia would increase the bandwidth of the system and enable potential high performance applications. Future work thus includes a redesign of the robotic arm towards a fully inflatable design.

\section{ACKNOWLEDGMENT}

The authors would like to thank Michael Egli, Daniel Wagner, Andrea Buono and Marc-Andrè Corzillius for their contribution to the development of the prototype presented.

\section{REFERENCES}

[1] B. Mosadegh, P. Polygerinos, C. Keplinger, S. Wennstedt, R. Shepherd, U. Gupta, J. Shim, K. Bertoldi, C. Walsh, and G. Whitesides, "Pneumatic networks for soft robotics that actuate rapidly," Advanced Functional Materials, vol. 24, no. 15, pp. 2163-2170, 2014.

[2] S. Voisembert, N. Mechbal, A. Riwan, and A. Aoussat, "Design of a novel long-range inflatable robotic arm: Manufacturing and numerical evaluation of the joints and actuation," ASME Journal of Mechanisms and Robotics, vol. 5, 2013.

[3] S. Li, D. M. Vogt, D. Rus, and R. J. Wood, "Fluid-driven origamiinspired artificial muscles," Proceedings of the National Academy of Sciences, 2017.

[4] H. K. Yap, F. Sebastian, C. Wiedeman, and C. H. Yeow, "Design and characterization of low-cost fabric-based flat pneumatic actuators for soft assistive glove application," in IEEE International Conference on Rehabilitation Robotics (ICORR), 2017, pp. 1465-1470.

[5] S. Sanan, M. H. Ornstein, and C. G. Atkeson, "Physical human interaction for an inflatable manipulator," in Annual International Conference of the IEEE Engineering in Medicine and Biology Society $(E M B C), 2011$, pp. 7401-7404.

[6] R. Qi, T. L. Lam, and Y. Xu, "Mechanical design and implementation of a soft inflatable robot arm for safe human-robot interaction," in IEEE International Conference on Robotics and Automation (ICRA), 2014, pp. 3490-3495.

[7] B. Gorissen, D. Reynaerts, S. Konishi, K. Yoshida, J. Kim, and M. De Volder, "Elastic inflatable actuators for soft robotic applications," Advanced Materials, vol. 29, no. 43, 2017.

[8] I. Gaiser, R. Wiegand, O. Ivlev, A. Andres, H. Breitwieser, S. Schulz, and G. Bretthauer, Compliant Robotics and Automation with Flexible Fluidic Actuators and Inflatable Structures. In Smart Actuation and Sensing Systems - Recent Advances and Future Challenges. InTech, 2012.

[9] M. T. Gillespie, C. M. Best, and M. D. Killpack, "Simultaneous position and stiffness control for an inflatable soft robot," in IEEE International Conference on Robotics and Automation (ICRA), 2016, pp. 1095-1101.

[10] H. Kim, A. Kawamura, Y. Nishioka, and S. Kawamura, "Mechanical design and control of inflatable robotic arms for high positioning accuracy," Advanced Robotics, vol. 32, no. 2, pp. 89-104, 2018.

[11] R. F. Natividad, M. R. D. Rosario, P. C. Y. Chen, and C. H. Yeow, "A hybrid plastic-fabric soft bending actuator with reconfigurable bending profiles," in IEEE International Conference on Robotics and Automation (ICRA), 2017, pp. 6700-6705.

[12] M. Jordan, D. Pietrusky, M. Mihajlov, and O. Ivlev, "Precise position and trajectory control of pneumatic soft-actuators for assistance robots and motion therapy devices," in IEEE International Conference on Rehabilitation Robotics (ICORR), 2009, pp. 663-668.

[13] Y. Nishioka, M. Uesu, H. Tsuboi, S. Kawamura, W. Masuda, T. Yasuda, and M. Yamano, "Development of a pneumatic soft actuator with pleated inflatable structures," Advanced Robotics, vol. 31, no. 14, pp. 753-762, 2017. 
[14] M. Kataoka, H. Kimura, and N. Inou, "Hermetically-sealed flexible mobile robot "MOLOOP" for narrow terrain exploration," in Annual Conference of the IEEE Industrial Electronics Society (IECON), 2013, pp. 4156-4161.

[15] S. Voisembert, A. Riwan, N. Mechbal, and A. Barraco, "A novel inflatable robot with constant and continuous volume," in IEEE International Conference on Robotics and Automation (ICRA), 2011, pp. 5843-5848.

[16] M. Mihajlov, M. Hubner, O. Ivlev, and A. Graser, "Modeling and control of fluidic robotic joints with natural compliance," in IEEE International Conference on Control Applications (CCA), 2006, pp. $2498-2503$.

[17] C. M. Best, J. P. Wilson, and M. D. Killpack, "Control of a pneumatically actuated, fully inflatable, fabric-based, humanoid robot," in IEEE International Conference on Humanoid Robots (Humanoids), 2015, pp. 1133-1140.

[18] D. Maruyama, H. Kimura, M. Koseki, and N. Inou, "Driving force and structural strength evaluation of a flexible mechanical system with a hydrostatic skeleton," Journal of Zhejiang University SCIENCE A, vol. 11 , no. 4, pp. 255-262, 2010.

[19] H. J. Kim, Y. Tanaka, A. Kawamura, S. Kawamura, and Y. Nishioka, "Improvement of position accuracy for inflatable robotic arm using visual feedback control method," in IEEE/ASME International Conference on Advanced Intelligent Mechatronics (AIM), 2015, pp. 767-772.

[20] J. Taghia, A. Wilkening, and O. Ivlev, "Position control of soft-robots with rotary-type pneumatic actuators," in ROBOTIK 2012, German Conference on Robotics, 2012, pp. 399-404.

[21] C. M. Best, M. T. Gillespie, P. Hyatt, L. Rupert, V. Sherrod, and M. D. Killpack, "A new soft robot control method: Using model predictive control for a pneumatically actuated humanoid," IEEE Robotics Automation Magazine, vol. 23, no. 3, pp. 75-84, 2016.

[22] B. Cazzolato and Z. Prime, "On the Dynamics of the Furuta Pendulum," Journal of Control Science and Engineering, vol. 2011.

[23] M. J. Troughton, Handbook of Plastics Joining, Second Edition: A Practical Guide (Plastics Design Library). William Andrew, 2008.

[24] E. Richer and Y. Hurmuzlu, "A high performance pneumatic force actuator system: Part 1 - nonlinear mathematical model," ASME Journal of Dynamic Systems, Measurement, and Control, vol. 122, no. 3, pp. 416-425, 1999.

[25] "Pneumatic fluid power. Determination of flow-rate characteristics of components using compressible fluids," International Organization for Standardization, Standard, 2013.

[26] M. Pipan and N. Herakovič, "Volume flow characterization of pwmcontrolled fast-switching pneumatic valves," Strojniški vestnik - Journal of Mechanical Engineering, vol. 62, no. 9, pp. 543-550, 2016.

[27] S. Boyd, "Multitone signals with low crest factor," IEEE Transactions on Circuits and Systems, vol. 33, no. 10, pp. 1018-1022, 1986.

[28] K. M. Moudgalya, Linear Quadratic Gaussian Control. John Wiley \& Sons, Ltd, 2007, pp. 463-485.

[29] M. Gäfvert, "Dynamic Model Based Friction Compensation on the Furuta Pendulum," in IEEE International Conference on Control Applications (CCA), 1999, pp. 1260-1265. 